\title{
Community Perceptions of Air Pollution and Related Health Risks in Nairobi Slums
}

Thaddaeus Egondi ${ }^{1,2, *}$, Catherine Kyobutungi ${ }^{1}$, Nawi $\mathrm{Ng}^{2}$, Kanyiva Muindi ${ }^{1,2}$, Samuel Oti ${ }^{1,3}$, Steven van de Vijver ${ }^{1,3}$, Remare Ettarh ${ }^{4}$ and Joacim Rocklöv ${ }^{2}$

1 African Population and Health Research Center, P.O. Box 10787, Nairobi 00100, Kenya; E-Mails: ckyobutungi@aphrc.org (C.K.); kmuindi@aphrc.org (K.M.); soti@aphrc.org (S.O.); svijver@gmail.com (S.V.)

2 Department of Public Health and Clinical Medicine, Epidemiology and Global Health, Umeå University, Umeå SE-901 85, Sweden; E-Mails: Nawi.Ng@epiph.umu.se (N.N.); joacim.rocklov@envmed.umu.se (J.R.)

3 Department of Global Health, Academic Medical Centre, University of Amsterdam, Amsterdam Institute for Global Health and Development, Amsterdam 1100 DE, The Netherlands

4 Faculty of Medicine, University of British Columbia, Vancouver, BC V6T 1Z3, Canada; E-Mail: rettarh@gmail.com

* Author to whom correspondence should be addressed; E-Mails: tegondi@aphrc.org or tegondi@yahoo.co.uk; Tel.: +254-(20)-400-1000; Fax: +254-(20)-400-1101.

Received: 1 July 2013; in revised form: 9 September 2013 / Accepted: 10 September 2013 / Published: 11 October 2013

\begin{abstract}
Air pollution is among the leading global risks for mortality and responsible for increasing risk for chronic diseases. Community perceptions on exposure are critical in determining people's response and acceptance of related policies. Therefore, understanding people' perception is critical in informing the design of appropriate intervention measures. The aim of this paper was to establish levels and associations between perceived pollution and health risk perception among slum residents. A cross-sectional study of 5,317 individuals aged 35+ years was conducted in two slums of Nairobi. Association of perceived score and individual characteristics was assessed using linear regression. Spatial variation in the perceived levels was determined through hot spot analysis using ArcGIS. The average perceived air pollution level was higher among residents in Viwandani compared to those in Korogocho. Perceived air pollution level was positively associated with perceived health risks. The majority of respondents were exposed to air pollution in
\end{abstract}


their place of work with $66 \%$ exposed to at least two sources of air pollution. Less than $20 \%$ of the respondents in both areas mentioned sources related to indoor pollution. The perceived air pollution level and related health risks in the study community were low among the residents indicating the need for promoting awareness on air pollution sources and related health risks.

Keywords: perceived air quality; air pollution; perceived health risk; urban slum

\section{Introduction}

Exposure to urban air pollution is one of several environmental and public health concerns currently confronting the World's population [1]. Several studies have established an association between air pollution and health effects [2-4]. Exposure to air pollution leads to adverse health effects ranging from respiratory illness to chronic illness such as cancer, adverse pregnancy outcomes and premature death. About 3.3 million premature deaths are attributed to both indoor and outdoor air pollution yearly and the burden is high among those living in middle-income countries [5]. Low socioeconomic status generally is associated with poor health, which makes people susceptible to the damaging effects of air pollution [6,7]. Moreover, people with low socioeconomic status such as those living in urban slums are more likely to live closer to roadways and polluting industrial facilities thus exposing them to greater levels of pollutants. They also have less access to health care thereby exacerbating any adverse health outcomes [6].

Sources of outdoor air pollution in urban slums are mainly dust, burning of trash, vehicle and industrial emissions. Due to poor ventilation in these settings, outdoor air pollutants infiltrate into households raising levels of indoor air pollution. This combination of indoor and outdoor air pollution increases the burden of air pollution in deprived urban areas. In developing countries, indoor air pollutants are mainly released during the combustion of solid fuels used for cooking and heating. Households using such fuels are generally located in poor communities in rural areas and urban slums with poorly ventilated houses [8]. Though several studies have shown the link between exposure to air pollution and health, most of these studies have been carried out in high-income settings. In addition, few studies have tried to understand the perceptions of residents about air pollution and related risks particularly among the very poor population.

Benefits of reducing exposure to air pollution are well documented from both natural experiments $[4,9,10]$ and epidemiological studies [11,12]. In an effort to reduce air pollution, most air quality management bodies have focused on the emissions-based control programs [13]. For example in Kenya, a draft air pollution regulation set up by the National Environmental Management Authority (NEMA) focuses on controlling the emissions [14]. Although regulations targeting emissions have led to a decrease in levels of pollution, interventions targeting individuals, which reduce exposure independent of emissions, will greatly mitigate health impacts of air pollution [13,15-17]. A recent study suggests a new framework of reducing air pollution-related health impacts that incorporates strategies at regulatory, community, and individual levels to reduce both emission and exposure [13]. 
Strategies targeting either the community or individual levels require contextual knowledge of the perceptions of both exposure and associated risk.

Perception is an important component of behavior change and plays a major role in public response to environmental exposures [18-20]. Therefore, increasing people's perception and knowledge is a cornerstone for interventions promoting protective behavior. This paper adopted the definition of perception by Sjöberg et al. as subjective assessment of exposure level to an environmental hazard and the concern with the consequences of the exposure [21]. Research on environmental risk assessment has established a relationship between exposure and health risks. However, little attention has been paid to understanding community perceptions of environmental risk particularly in sub-Saharan Africa. Consequently, governments are grappling with how to empower citizens to be involved in various aspects of environmental management to increase action and local participation in interventions [22].

Furthermore, community and individual level interventions for reducing exposure to air pollution are important means for improving public health and citizen participation. However, the effectiveness of these programs depends mainly on peoples' perceptions of exposure and risk for individual acceptance and action. Studies on risk perception have revealed it to be multi-dimensional with demographic, cultural and political characteristics playing a role in observed differences in perception.

Annoyance has also been identified as one consequence of air pollution [23] and is said to be a useful signal for potential health effects of pollution in a community [24]. Studies have found that annoyance involves individual perceptions and attitudes towards the exposure [23] influenced by factors such as sex, age and prior exposure to the pollutant [25]. Understanding perception and attitudes, including annoyance, of the public towards air quality and related health risks is therefore critical in informing the design of intervention programs as well as for successful individual involvement in the mitigation process. Interventions directed at individuals have also been suggested to lower baseline health risks and overall burden of diseases associated with air pollution $[13,26]$. Such interventions include, but are not limited to, control of hypertension and smoking cessation.

\section{Study Context}

The study is set in two informal settlements or slums (Korogocho and Viwandani) located in Nairobi, the capital of Kenya. The two slums form part of the Nairobi Urban Health and Demographic Surveillance System (NUHDSS) run by African Population and Health Research Center (APHRC). Low household incomes, low levels of education, and high proportions of the population employed in manual/unskilled occupations typify both slums. Both areas are characterized by unpaved roads, open burning of refuse and cooking along the road using sawdust or sacks made from sisal or cotton/plastic materials. There are refuse-dumping sites located close to each of these study areas and specifically, the largest refuse dump site in Nairobi is situated to the east and south east of the Korogocho slum settlement. Smoke from these dumping sites bellows out daily, day and night, and even during the rainy season. Viwandani, one of the informal settlements, is located along a line of known polluting industries in the city's industrial area. Despite lack of actual measurements of air pollution, these characteristics suggest that it is an environmentally disadvantaged neighborhood in terms of air pollution.

Work on the burden of disease in the informal settlements of Nairobi indicates high prevalence of respiratory illness, asthma [27], and acute respiratory infections as the leading contributor to the 
mortality burden [28] among children. Studies in the two areas have also shown seasonal mortality among under-five children with a high peak during the cold season [29-31]. It is postulated that high mortality during the cold season may be due to increase in level of indoor pollution as a result of people trying to keep warm using solid fuels. Despite the apparent numerous sources of both outdoor and indoor air pollution, it remains unclear to what extent slum residents perceive air pollution as a major problem for their health. Therefore, the aims of this study were to establish perceived air pollution levels, health risk and annoyance; investigate the socio-demographic factors associated with perceived air pollution levels and related health risk, and determine the spatial variation in the perceived level of pollution and health risk among slum residents in Nairobi.

\section{Methods}

\subsection{Study Design and Procedures}

Data collection for this study was nested in a larger interventional study within the NUHDSS known as the Sustainable model for Cardiovascular Health by Adjusting Lifestyle and Treatment with Economic Perspective in settings of Urban Poverty (SCALE UP) study [32]. The target sample for the SCALE UP study included adults aged 35 years and above living in the two slums of Korogocho and Viwandani that gave informed consent to participate in that study. For its own purposes, participants in the SCALE UP study were recruited through different methods in either slum. In Korogocho, it was done through a census of all adults older than 35 years while in Viwandani, it was done through a random sample of similarly aged residents. The different sampling methods in two areas are not expected to affect the results since the random sample was a representative sample of Viwandani. The basis for the recruitment was the sampling frame of the NUHDSS which has a complete listing of all households and individuals within the Demographic Surveillance Area of the two settlements and is described in detail elsewhere [33].

Our study leveraged the data collection platform of the SCALE UP study to administer our own data collection tools to each consenting participant in both slums. The informed consent form clearly explained to the participants that both studies had separate objectives and elaborated on what these objectives were. Data were collected by trained interviewers who administered a structured questionnaire to participants. The questionnaire asked participants about their perception of air quality, air pollution-related health effects, annoyance with air pollution and sources of information on air pollution in addition to individual characteristics.

\subsection{Description of Measures}

The questionnaire included several questions on air pollution level, perceived associated health risk and annoyance from air pollution as summarized in Table 1. The questions included options of yes/no and a five-point ordinal scale. To measure level of annoyance, the respondents were asked to assume that people's level of annoyance due to indoor and outdoor air pollution from any source could be stacked on a ladder or staircases of five steps, with low level (1) representing "No Annoyance" and high level (5) representing "Extreme Annoyance". Then respondents were asked to place themselves on the ladder that corresponds to their level of annoyance due to outdoor or indoor air pollution. 
The questions were adopted from studies that used similar scales [34,35] though we reduced scales from 11-point scales to 5-point scales. The composite measures were generated using the alpha STATA command. This command standardizes each variable to a scale of mean 0 and variance of 1 before combining them into a single score. The final composite measure was obtained by averaging standardized individual items for each participant. The standardized scores generated were then transformed to a scale of 0 to 100 for ease of interpretation. The approach allows combining questions with different response scales. High scores represent perceived high level of air pollution or perceived high health risk associated with air pollution.

Table 1. List of questions for the composite indices of perceived level of pollution and perceived related health risk.

\section{Perceived air pollution}

How would you rate the quality of air in the community where you live (Viwandani/Korogocho)? Would you say it is (Very Low, Low, High, Very High)

How would you rate the quality of air in your house? Options (Very Low, Low, High, Very High)

Which of the following would you say are the sources of outdoor or indoor air pollution within Korogocho/Viwandani (Dust, Vehicle emissions, Industrial emissions, Cooking fuels, Burning trash, Smelly sewage, Cigarette smoking, Other sources)? Options (Yes or No)

How severe would you say is air pollution in Korogocho/Viwandani from (Dust, Vehicle emissions, Industrial emissions, Cooking fuels, Burning trash, Smelly sewage, Cigarette smoking, Other sources)? Options (None, Low, Moderate, High and Very High)

\section{Perceived health risks}

How much health risk do you think each of the following poses to you and your family (dust, vehicle emissions, industrial emissions, cooking fuels, burning trash, smelly sewage, cigarette smoking, and other sources)? Options (None, Low, Moderate, High and Very High)

What health problems do you think are brought about by air pollution (cough/cold, difficulty breathing, eye problems, asthma, cancer, heart problems, headache, other)? Options (Yes/No)

Individual characteristic variables included sex, age, and duration of stay in the community, marital status, education level and current occupation status. Occupation status was grouped into four main categories namely those involved in business activities, informal employment, formal employment and those not working or involved in agricultural activities. The variable daily work locations exposed to air pollution was defined as the number of daily work locations (next to busy roads, near cooking places, dusty place, and factory) mentioned that are considered highly polluted areas. The variable of information on air pollution used for regression analysis was defined as either heard or never heard any information on air pollution. 


\subsection{Statistical Analysis Approach}

The responses to different questions were summarized in terms of proportions by the two study sites. The composite measures were summarized in terms of averages by demographic characteristics to assess the distribution of the perception levels across key characteristics. The differences or association of composite measures with different characteristics were assessed using linear regression analysis. Regression analysis was done in two steps. First, bivariate analysis was performed to assess the association of each factor with perceived level of pollution or health risk independently. The purpose of bivariate analysis was to determine the independent relationship between each of the characteristics and the outcome without controlling for others. Secondly, multiple regression analysis was conducted to assess the association of different factors controlling for the other factors. Regression assumptions were assessed using graphical inspection of residuals from a multiple regression model plotted against fitted values.

Spatial variability in the perceived level of air pollution and related health risks was assessed and visualized using hot spot analysis in ArcGIS. The hot spot analysis tool calculates the Getis-Ord statistic for each geo-referenced household to identify where those households with either high or low attribute values cluster spatially. The analysis looks at each household within the context of neighboring households. A household is considered a statistically significant hot spot if it has a high value and is surrounded by other features with high values as well. The same applies for the households classified as cold spots. In this study, hot spots are areas of households of people with high perceived level of air pollution whereas cold spots are of those households of people with low perceived level of air pollution.

\section{Results}

\subsection{Description of the Sample}

The study was conducted from August to December 2012 and a total sample of 5,317 residents was interviewed (3,887 in Korogocho and 1,430 in Viwandani). The sample distribution by socio-demographic characteristics is summarized in Table 2. In Viwandani, there were more male respondents and this is because the area attracts mainly the population seeking employment in the industries. The two study areas differed in age distribution with Viwandani consisting of younger population compared to Korogocho. About $49 \%$ of the residents in Korogocho had lived there for at least 20 years compared to about 16\% in Viwandani. Most respondents were married in both study areas with high proportion of respondents not married in Viwandani (30\%) compared to Korogocho (15\%). The proportion of respondents who never went to school was higher in Korogocho (17\%) as compared to Viwandani (4\%) and very few respondents had attained college or higher level of education (3\% in Viwandani and $1 \%$ in Korogocho). The two main occupations of the residents in the two sites were informal business and casual labor. 
Table 2. Percentage distribution of the study sample by the two study sites.

\begin{tabular}{|c|c|c|}
\hline & Viwandani & Korogocho \\
\hline & $(\mathrm{n}=\mathbf{1 , 4 3 0})$ & $(\mathrm{n}=\mathbf{3 , 8 8 7})$ \\
\hline \multicolumn{3}{|c|}{$\operatorname{Sex}\left(\chi^{2}(1)=71.6 ; p\right.$-value $\left.=0.000\right)$} \\
\hline Female & 36.1 & 49.1 \\
\hline Male & 63.9 & 50.9 \\
\hline \multicolumn{3}{|c|}{ Respondent's Age $\left(\chi^{2}(3)=238.9 ; p\right.$-value $\left.=0.000\right)$} \\
\hline $35-40$ years & 41.9 & 26.3 \\
\hline $41-50$ years & 41.5 & 37.7 \\
\hline $51-60$ years & 13.3 & 21.4 \\
\hline $60+$ years & 3.3 & 14.6 \\
\hline \multicolumn{3}{|c|}{ Duration of stay $\left(\chi^{2}(3)=595.7 ; p\right.$-value $\left.=0.000\right)$} \\
\hline 0-10 years & 41.0 & 17.5 \\
\hline $11-20$ years & 43.3 & 33.9 \\
\hline 21-30 years & 14.4 & 30.9 \\
\hline $30+$ years & 1.2 & 17.8 \\
\hline \multicolumn{3}{|c|}{ Marital status $\left(\chi^{2}(1)=119.2 ; p\right.$-value $\left.=0.000\right)$} \\
\hline Married & 85.3 & 70.5 \\
\hline Not married & 14.7 & 29.5 \\
\hline \multicolumn{3}{|c|}{ Education level $\left(\chi^{2}(2)=343.5 ; p\right.$-value $\left.=0.000\right)$} \\
\hline Less than primary & 15.2 & 38.0 \\
\hline Primary & 50.8 & 46.2 \\
\hline Secondary+ & 34.0 & 15.8 \\
\hline \multicolumn{3}{|c|}{ Current Occupation $\left(\chi^{2}(3)=662.9 ; p\right.$-value $\left.=0.000\right)$} \\
\hline Business & 29.4 & 43.0 \\
\hline Informal & 35.1 & 34.5 \\
\hline Formal & 27.0 & 3.9 \\
\hline Other & 8.6 & 18.6 \\
\hline
\end{tabular}

\subsection{Location of Work, Sources of Pollution, Health risks and Information on Air Pollution}

The findings regarding location of work, health risks, sources of pollution and information on air pollution are summarized in Table 3. Majority of respondents in both study areas reported that their daily work is located next to either a busy road or in a dusty place. In both sites, about half of the respondents reported working near a place where cooking takes place. In Viwandani: 13\% of respondents reported working in all the four areas; next to a busy road, in a dusty place, near a place where cooking is taking place and in a factory where air is bad compared to only $3 \%$ in Korogocho. In both locations, most of respondents (81\%) reported smelly trenches as the source of air pollution, although this is not scientifically considered a source of air pollution. Dust and burning of trash was mentioned as source of air pollution, more often among the respondents in Korogocho than those in Viwandani. Industrial emissions were reported to be a major source of pollution in Viwandani (55\%) compared with only 5\% in Korogocho. Vehicles were mentioned as a source of pollution by more respondents in Korogocho (20\%) compared to Viwandani (7\%). 
Table 3. The percentage distribution of respondents' location of work, perceived sources of air pollution and health risks, and sources of information on air pollution by study site.

\begin{tabular}{|c|c|c|c|}
\hline & Viwandani & Korogocho & Total \\
\hline & $\%$ & $\%$ & $p$-value \\
\hline \multicolumn{4}{|l|}{ Location of respondent's daily work } \\
\hline Next to a busy road & 72.0 & 68.1 & 0.018 \\
\hline Near a place where cooking takes place & 46.9 & 52.8 & 0.001 \\
\hline In a dusty place & 70.2 & 68.4 & 0.260 \\
\hline In a factory where air is bad & 40.6 & 10.4 & $<0.001$ \\
\hline Not in a fixed place & 56.4 & 38.1 & $<0.001$ \\
\hline At least one of the above & 71.3 & 68.6 & 0.058 \\
\hline \multicolumn{4}{|l|}{ Perceived sources of air pollution } \\
\hline \multicolumn{4}{|l|}{ Outdoor air pollution sources } \\
\hline Dust & 47.5 & 63.0 & $<0.001$ \\
\hline Vehicles & 7.7 & 20.3 & $<0.001$ \\
\hline Industries & 55.5 & 5.0 & $<0.001$ \\
\hline Burning of Trash & 50.5 & 66.6 & $<0.001$ \\
\hline \multicolumn{4}{|l|}{ Indoor air pollution sources } \\
\hline Cooking fuels & 13.8 & 22.1 & $<0.001$ \\
\hline Cigarette Smoking & 11.6 & 18.5 & $<0.001$ \\
\hline \multicolumn{4}{|l|}{ Other } \\
\hline Smelling of Trenches & 81.1 & 81.8 & 0.568 \\
\hline Other sources & 9.5 & 13.1 & $<0.001$ \\
\hline \multicolumn{4}{|l|}{ Perceived health risks from air pollution } \\
\hline Cough/Cold & 61.5 & 74.4 & $<0.001$ \\
\hline Difficulty breathing & 39.9 & 46.4 & $<0.001$ \\
\hline Eye problem & 15.2 & 27.7 & $<0.001$ \\
\hline Asthma & 10.2 & 14.7 & $<0.001$ \\
\hline Cancer & 6.4 & 6.5 & 0.904 \\
\hline Heart problem & 15.1 & 13.3 & 0.089 \\
\hline Headache & 16.5 & 30.1 & $<0.001$ \\
\hline Don't know & 10.2 & 6.3 & $<0.001$ \\
\hline OTHER health risks & 24.5 & 17.6 & $<0.001$ \\
\hline \multicolumn{4}{|l|}{ Sources of information on air pollution } \\
\hline Radio & 32.3 & 39.1 & $<0.001$ \\
\hline TV & 12.5 & 14.6 & 0.059 \\
\hline Newspapers & 5.7 & 6.1 & 0.629 \\
\hline Barazas (Community meetings) & 5.4 & 18.4 & $<0.001$ \\
\hline Health workers/facilities & 9.7 & 25.1 & $<0.001$ \\
\hline Never heard & 55.0 & 35.1 & $<0.001$ \\
\hline
\end{tabular}

The two main sources of indoor air pollution being cooking fuels and cigarette smoking were among the least reported source of air pollution in Viwandani. Cough/cold, difficulties in breathing, headache and eye problems were the most common health risks mentioned related to air pollution. Only $10 \%$ of the respondents in Viwandani and 6\% in Korogocho did not know of any health problems related to air pollution. Radio was reported as main source of information regarding air pollution 
though 55\% of respondents in Viwandani and 35\% in Korogocho had never received any information related to air pollution.

\subsection{Factors Related to Community Perceptions on Air Pollution}

The average score of perceived air pollution level was 46.9 (95\% CI $=46.0$ to 47.8 ) in Viwandani and $41.4(95 \% \mathrm{CI}=40.9$ to 41.9$)$ in Korogocho. The average score for perceived level of health risk related to air pollution was $43.6(95 \% \mathrm{CI}=42.7$ to 44.5$)$ in Viwandani and $44.6(95 \% \mathrm{CI}=44.1$ to 45.1) in Korogocho. Descriptive summary statistics for the perceived scores are presented in Table 4.

Table 4. Descriptive summary of the perceived scores by individual characteristics.

\begin{tabular}{|c|c|c|c|}
\hline & $\begin{array}{c}\text { Air pollution } \\
\text { perceived score } \\
\text { Mean (SD) }\end{array}$ & $\begin{array}{c}\text { Health risk } \\
\text { perceived score } \\
\text { Mean (SD) }\end{array}$ & $\begin{array}{l}\text { Annoyance } \\
\text { Mean (SD) }\end{array}$ \\
\hline \multicolumn{4}{|l|}{ Study Site } \\
\hline Viwandani & $46.9(17.3)$ & $43.6(17.4)$ & $41.7(18.8)$ \\
\hline Korogocho & $41.4(15.8)$ & $44.6(16.6)$ & $48.3(18.2)$ \\
\hline \multicolumn{4}{|l|}{$\operatorname{Sex}$} \\
\hline Female & $42.5(16.1)$ & $44.1(16.9)$ & $46.8(18.6)$ \\
\hline Male & $43.2(16.7)$ & $44.5(16.8)$ & $46.2(18.7)$ \\
\hline \multicolumn{4}{|l|}{ Respondent's Age } \\
\hline $35-40$ years & $44.1(17.0)$ & $44.8(16.7)$ & $46.3(18.3)$ \\
\hline $41-50$ years & $43.1(16.4)$ & $44.5(16.9)$ & $46.6(18.9)$ \\
\hline 51-60 years & $41.8(16.0)$ & $44.0(16.7)$ & $46.7(18.7)$ \\
\hline $60+$ years & $40.7(15.5)$ & $43.1(17.0)$ & $46.4(18.3)$ \\
\hline \multicolumn{4}{|l|}{ Duration of Stay } \\
\hline $0-10$ years & $44.3(16.8)$ & $44.1(16.5)$ & $45.6(18.9)$ \\
\hline $11-20$ years & $43.2(16.8)$ & $44.4(17.2)$ & $46.8(18.2)$ \\
\hline $21-30$ years & $42.4(15.8)$ & $44.1(16.5)$ & $46.4(18.6)$ \\
\hline $30+$ years & $40.6(15.6)$ & $45.1(17.0)$ & $47.5(19.2)$ \\
\hline \multicolumn{4}{|l|}{ Marital status } \\
\hline Married & $42.2(15.7)$ & $43.8(16.4)$ & $47.3(18.4)$ \\
\hline Not married & $43.3(16.5)$ & $44.7(16.7)$ & $46.2(18.7)$ \\
\hline \multicolumn{4}{|l|}{ Education Level } \\
\hline Less than Primary & $40.9(15.2)$ & $42.7(17.2)$ & $45.5(19.1)$ \\
\hline Primary & $43.8(16.7)$ & $45.5(16.3)$ & $47.1(18.0)$ \\
\hline Secondary+ & $44.4(16.9)$ & $45.0(16.5)$ & $46.6(19.2)$ \\
\hline \multicolumn{4}{|l|}{ Current Occupation } \\
\hline Business & $42.2(16.3)$ & $45.1(16.4)$ & $46.8(18.2)$ \\
\hline Informal & $44.0(16.6)$ & $45.4(16.6)$ & $47.2(18.7)$ \\
\hline Formal & $44.2(16.4)$ & $41.1(16.4)$ & $43.1(19.2)$ \\
\hline Other & $42.2(15.9)$ & $43.0(17.1)$ & $46.4(19.0)$ \\
\hline Total & $42.9(16.4)$ & $44.3(16.8)$ & $46.5(18.6)$ \\
\hline
\end{tabular}


At bivariate analysis, perceived air pollution was associated with duration of stay in the slum, age of the respondent, education level, occupation, received information on air pollution, number of work locations exposed to air pollution and slum of residence. Respondents who had stayed longer in the study area or those who were older perceived low level of air pollution when considered independently. However, this association was not significant after controlling for other factors. Residents in Korogocho had perceptions of low air pollution level compared to residents in Viwandani in the bivariate analysis. Therefore, final regression analysis for both perceived level of air pollution and health risk was performed for each slum of residence separately.

The results of the multiple linear regressions by study site are shown in Table 5. Duration of stay, age, gender and marital status were not significantly associated with perceived air pollution level in analysis stratified by study site.

Table 5. Characteristics associated with perceived level of air pollution and related health risks.

\begin{tabular}{|c|c|c|c|c|}
\hline & \multicolumn{2}{|c|}{ Air pollution } & \multicolumn{2}{|c|}{ Health Risk } \\
\hline & Viwandani & Korogocho & Viwandani & Korogocho \\
\hline Perceived pollution level & & & $0.44 *$ & $0.48 *$ \\
\hline \multicolumn{5}{|l|}{ Duration stay (ref: -10 years) } \\
\hline $11-20$ years & -1.16 & 0.63 & 1.32 & -0.47 \\
\hline $21-30$ years & -2.17 & 0.77 & 2.13 & -0.96 \\
\hline $30+$ years & -3.70 & 0.09 & 3.14 & 1.47 \\
\hline \multicolumn{5}{|l|}{ Age (ref: $35-40$ years) } \\
\hline $41-50$ years & 0.46 & -0.54 & -0.27 & 0.20 \\
\hline $51-60$ years & 1.11 & -1.37 & 0.28 & -0.11 \\
\hline $60+$ years & 4.43 & -1.46 & 2.21 & 0.20 \\
\hline Male & -0.23 & -0.85 & -1.86 & 0.30 \\
\hline Married (ref: Not married) & 0.49 & -0.20 & 0.21 & $1.09 *$ \\
\hline \multicolumn{5}{|l|}{ Education (ref: No education) } \\
\hline Primary & $3.80 *$ & 0.80 & -0.78 & $1.60 *$ \\
\hline Secondary + & $3.44 *$ & 0.38 & -0.71 & 1.29 \\
\hline \multicolumn{5}{|l|}{ Occupation (ref: Business) } \\
\hline Informal & -0.50 & $1.25 *$ & -0.87 & -0.89 \\
\hline Formal & $-3.20 *$ & $4.88 *$ & -1.26 & -1.87 \\
\hline Other/None & 2.42 & $5.12 *$ & $3.85 *$ & $-1.50 *$ \\
\hline Never heard any information & $2.31 *$ & $-2.19 *$ & $-5.06 *$ & $-1.4 *$ \\
\hline Number of polluted work locations & $9.61 *$ & $5.40 *$ & $10.05 *$ & 0.97 \\
\hline Annoyance level & $0.38 *$ & $0.07 *$ & $0.07 *$ & $0.21 *$ \\
\hline
\end{tabular}

* Statistically significant at 5\% level and the values represent linear regression coefficients.

Individuals with at least primary level of education perceived higher levels of air pollution compared to those with no or less than primary level. However, the relationship between education and perceived air pollution level was statistically significant only among residents of Viwandani. Occupation was significantly associated with perceived air pollution level and the relationship varied by the study site. In Viwandani, people engaged in formal employment perceived lower levels of air pollution and the perception of those in informal or other forms of occupations were not significant 
compared to those in business. Those in informal, formal and other forms of employment perceived higher levels of air pollution compared to those in business among Korogocho residents. Respondents who never heard information on air pollution significantly perceived lower levels of air pollution in Korogocho. Surprisingly, persons who never heard air pollution information in Viwandani perceived higher levels of air pollution compared to those who had. Exposure to working environments with air pollution was positively associated with perceived air pollution level. Perceived air pollution level was also positively associated with annoyance level among residents in both areas.

Perceived level of health risk was positively associated with perceived air pollution level in both areas. Duration of stay, age and gender were not significantly associated with perceived health risk related to air pollution after controlling for other individual characteristics. Perceived health risk was associated with marital status, education level, and occupation in multivariate analysis. Married people in Korogocho had a higher perceived health risk. Education was significantly associated with high perceived health risk in Korogocho. Other or no occupation was associated with perceived high health risk among residents in Viwandani and low perceived health risk among Korogocho residents. Those who had not heard information about air pollution were associated with low perceived risk in both areas. A similar positive association of highly perceived level of air pollution with exposure to working environments with air pollution and annoyance from air pollution was observed for perceived health risks.

Outdoor air quality was considered poor compared to indoor air quality. A majority of residents felt that the quality of indoor air was moderate or good and were mostly not annoyed by it. Annoyance level about outdoor air quality may directly relate to the perceived air pollution level. The variation in perceived air quality and annoyance level is illustrated in Figure 1 for Korogocho and Viwandani settlements, respectively.

Figure 1. The percentage rating of air quality and the level of annoyance from air pollution for both outdoor and indoor environments.

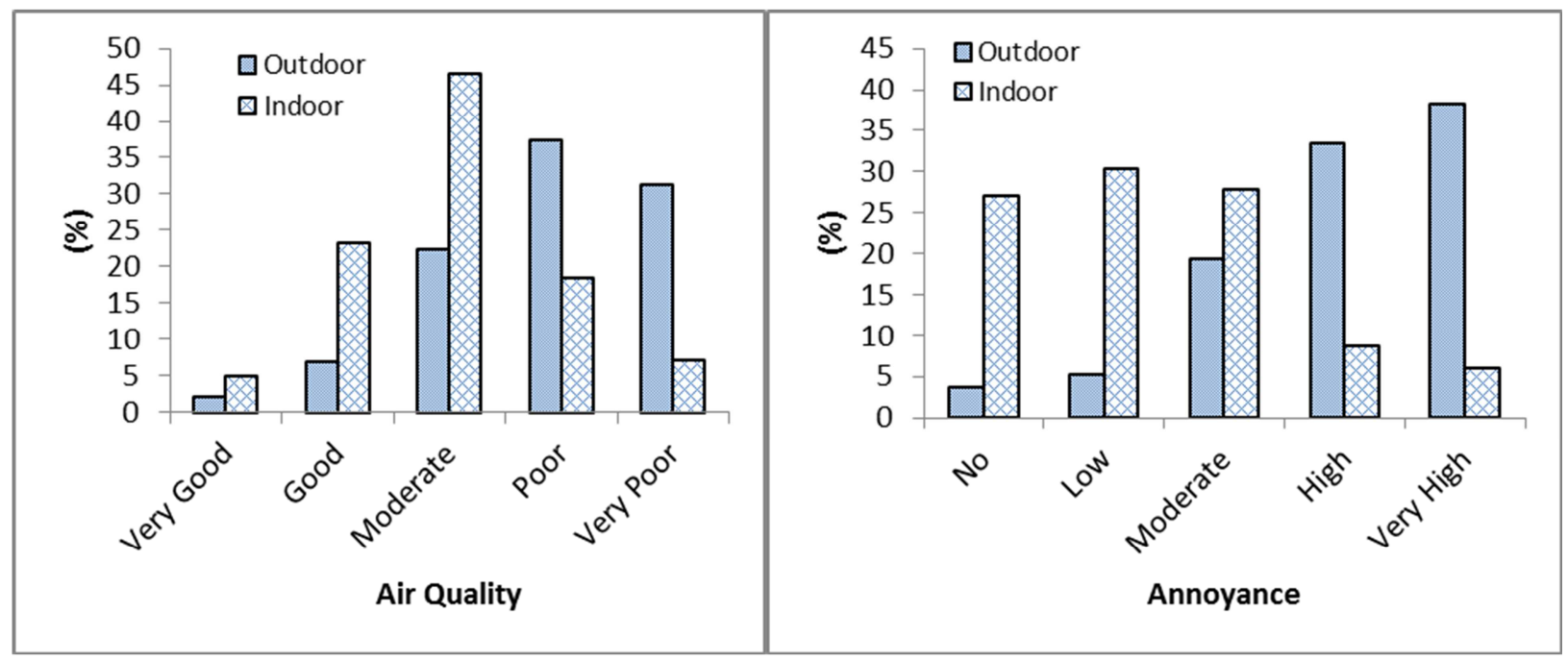




\subsection{Spatial Variation in Perceptions}

Figures 2 and 3 illustrate the distribution of perceived air pollution levels by village across the slums. The darker orange color represents areas in which respondents believe the local air pollution level was high.

Figure 2. Perceptions on the level of air pollution and related health risks across villages in Korogocho.

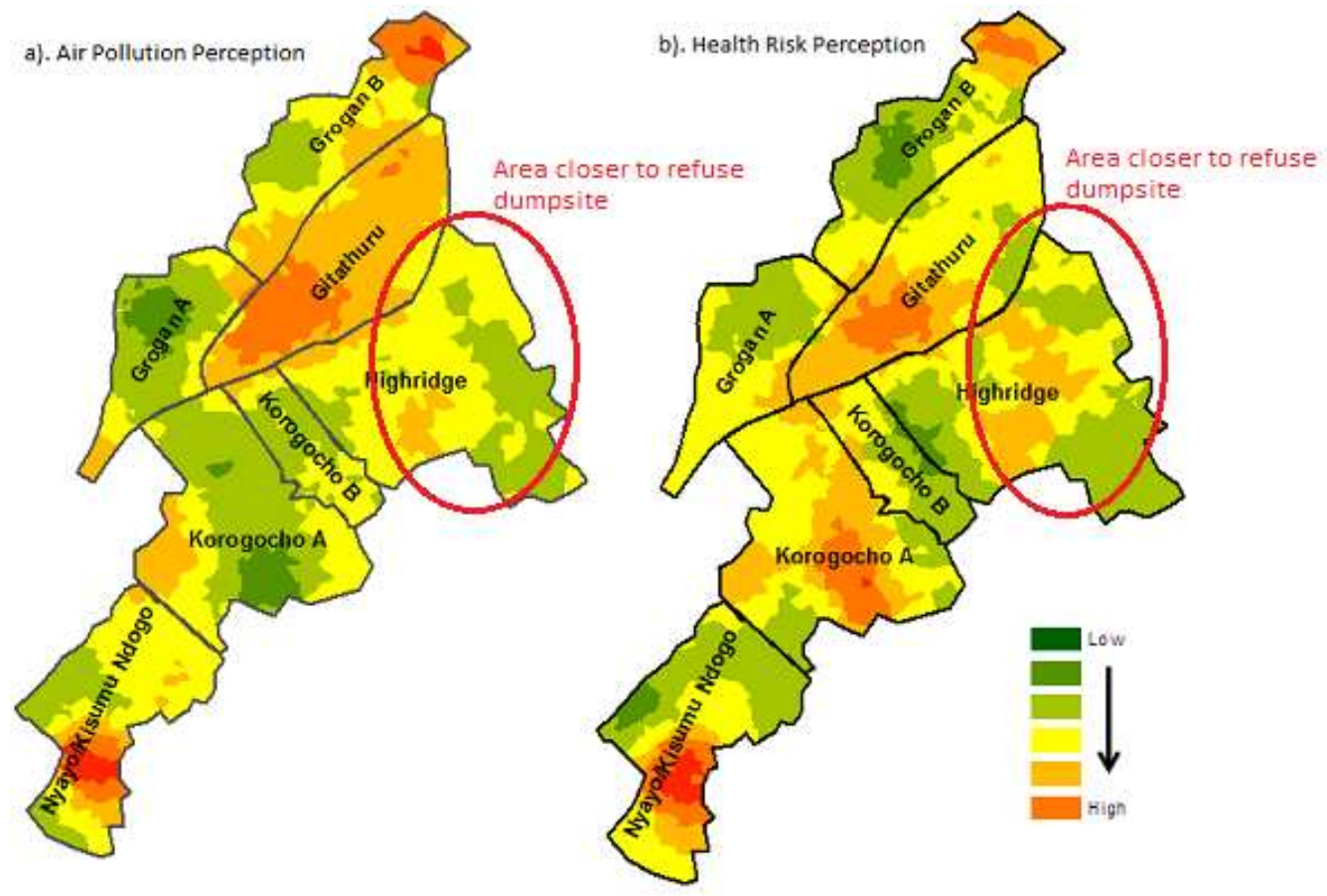

Figure 3. Perceptions on the level of air pollution and related health risks across villages in Viwandani.

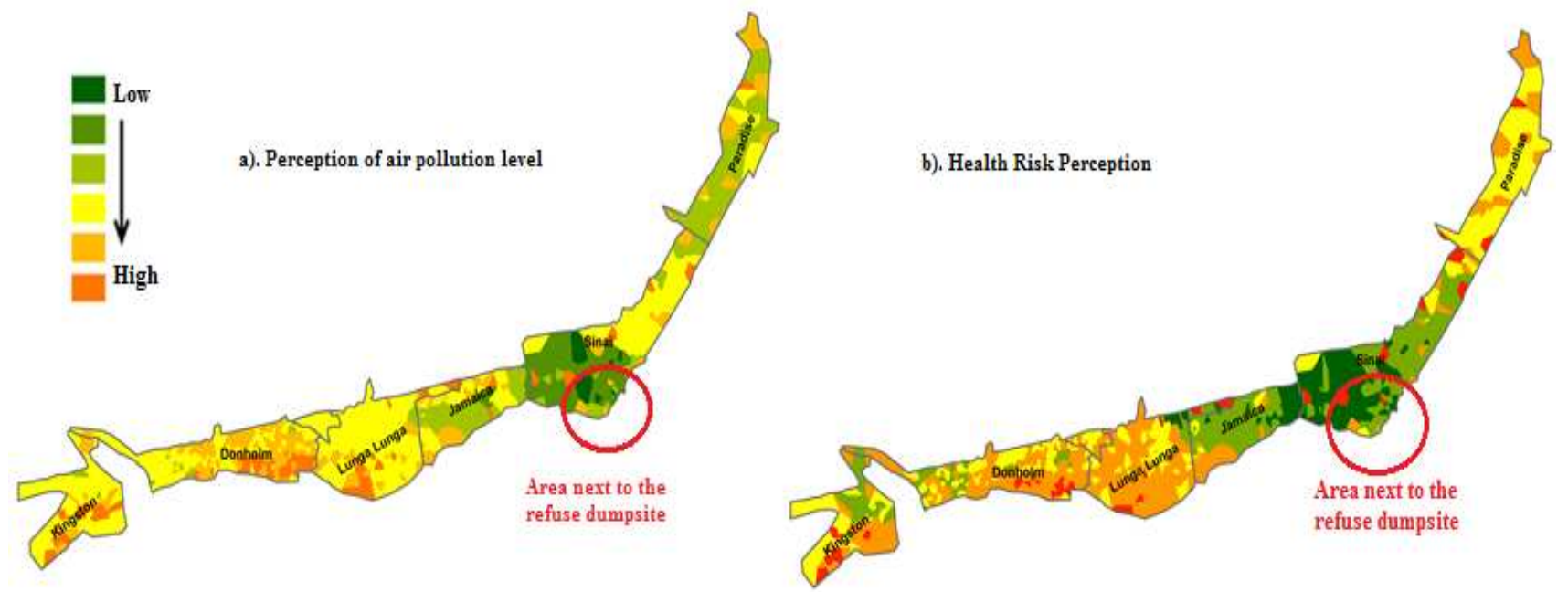

The general spatial pattern of poor air quality perceptions is concentrated in some specific areas similar to perceived health risks in both areas. The spatial pattern particularly in Korogocho seems to be influenced by the residents' activities and not the location of major sources of air pollution. For example, respondents closer to a major refuse dumpsite (Highridge village) had low knowledge of air pollution 
despite the constant bellowing of smoke from the dumpsite. A heterogeneous pattern of air quality perceptions was observed in all villages of both slums, representing a spatial mix of views on environmental quality. The spatial patterns observed for both air quality and health risks in the two slums indicate that perceptions of health risks are associated with the perceived air pollution level.

As stated above, Viwandani has a similar spatial pattern of local air quality perceptions where those who expressed high air pollution level were located within villages closer to industries believed to emit pollutants in the air. For example, residents closer to the paper industry in Donholm village believed there was high air pollution level (poor air quality). Poor drainage of wastes from industries particularly in Kingstone and Lunga Lunga areas also made the residents perceive high levels of air pollution in the area. In the central area (Sinai village), residents had low perception of poor air quality despite being closer to a dumpsite, which emits heavy smoke from open fires. The industries close to this area (Sinai) are construction and food production industries that are believed not to emit pollutants in the air as compared to other areas. Thus low perceived air pollution of the residents in this area might be based on this kind of belief. The burning of tires in Paradise village also raises concerns of air quality by the residents. Compared to Korogocho, the patches of perception for Viwandani are clearly distinct though there is heterogeneity with respect to perceptions in both slums. These results illustrated in the two figures also indicate that perceived levels of air pollution were higher in Viwandani compared to Korogocho.

\section{Discussion}

The main goal of this study was to examine perceived air quality, related health risks and associated individual characteristics using a regression analysis. The study also examined spatial variation of the perceived air quality and related risk using spatial analysis. The key findings of this study are as follows; That there is very poor perception of actual sources of air pollution levels and misconception of what ordinarily are not air pollution sources such as "smelly trenches" among residents in the two communities, there is poor perception on the magnitude and importance of indoor air pollution. The majority of the residents also expressed that they were exposed to air pollution in their workplaces.

We found that those respondents who perceived high levels of air pollution in their local community had at least primary level of education or were likely to be in formal employment among residents of the Viwandani area. The difference in the observed relationship between perception and individual characteristics in the two study areas can be explained by the difference in the activities taking place. Among major contributors of air pollution, residents mentioned dust, burning of trash and industries though they were more concerned with smelling of trenches as a result of poor drainage. We also found that there was considerable concern about cooking fuels as source of pollution but few people felt that vehicles were source of air pollution in the two study areas. However, research findings from other settings have shown that people largely perceive automobiles and industries as the major source of pollution [36-38].

Several studies have explored the association between respondents' perceptions and individual characteristics. The relationship between level of education and air quality perception or awareness remains controversial. We found that respondents with at least primary level of education were more concerned about air quality than those with no or less than primary level in Viwandani and the 
relationship was not significant in Korogocho. Previous studies have reported higher levels of education to be associated with higher annoyance level or poor air quality perceptions $[7,39]$. Contrary, some studies indicate that a low education level was a significant determinant of annoyance with perceived air pollution level [40,41], a finding that could have been confounded by the place of residence of these people. Socio-economic variables have been shown to be correlated with the perception of local air quality, suggesting that these may be important factors in a study of perceived air quality [42]. In our study, we found that involvement in different forms of occupation was associated with varying perception of poor air quality depending on the location. The importance of socioeconomic indicators in the context of air pollution research has been emphasized because they represent underlying aspects that affect susceptibility, exposure, or disease diagnosis and treatment [6]. Therefore, there is need for careful choice and interpretation of socioeconomic indicators depending on the location.

Though we did not find a significant association between age and perceptions of air quality, a study about the risks of greatest concern found that young respondents were more concerned about environmental issues, while older respondents were more likely to emphasize health and safety [43] while in another study it was found that young people aged 20-34 years had poor air quality perceptions compared to older age groups [7]. Lack of significant association between age and perceived air quality may be due to our study limitation of including only residents aged 35 and above. We did not find significant association between duration of stay in the study area and perceived air quality after adjusting for other individual characteristics.

In our study, we found that marital status was not associated with perceived air quality though there is relatively little written about any relation between marital status and perception of air quality and environmental issues. However, we observed significant association between marital status and high-perceived health risk in Korogocho. In his study, Kim et al. [7] found that ever married people are likely to perceive air quality as poor. Though explanation was not provided for this finding, in literature it is explained that married people may be more concerned about environmental pollution because spouses may be an important influence or source of information on environmental issues [44].

After adjusting for individual characteristics, we observed significant association between perceived air quality and perceived health risks related to air pollution. Previous studies reported a significant association between perceived air quality and self-reported health status $[45,46]$. Though self-reported health and perceived health risk refers to different concepts. Perceived health risk related to air pollution was found to be associated with level of education and the type of occupation though it varied by study location. Those in formal employment perceived low health risk as compared to those involved in business among Viwandani residence while opposite was observed in Korogocho. The fact that most business activities in the slum settlements are mainly informal and carried out along the roads or areas that are associated with high level of pollution can explain this observation. The difference between the two sites could be explained by the difference in the education levels of the residents. There were more people with no education in Korogocho as compared to Viwandani.

It is important to note that air quality perceptions mark differences in the two slums which indicates that perceptions in general may depend on an area's overall setting and availability of industries, other pollution sources or daily activities. For example, an important result from this study was that respondents living in villages closer to industries or poor drainage were more likely to perceive the air 
as more polluted than those living in other areas. Nevertheless, it is of concern that residents live in close proximity to some sources of pollution such as refuse dumpsite in both areas and yet do not perceive air pollution as a problem. Literature on perceptions of pollution has shown that people tend to attribute pollution to other areas away from their place of residence $[1,42]$. This could be the same tendency noted among residents of the villages next to known pollution sources; clearly showing the "halo effect". The use of GIS to map and spatially analyze the differences of air quality perceptions across the two slums helps in forming a clearer picture of how locations influence perceptions of environmental conditions.

\section{Conclusions}

The results of our study may help policymakers understand the need for education programs aimed at making residents in local neighborhoods aware of sources of air pollution and related health risks. The environmental management authorities need to direct effort on risk communication strategies to motivate personal direct perception or awareness of an environmental problem such as air pollution. This approach enhances the individual's understanding of the importance of environmental policy measures, which makes such measures easier to accept by community residents [47] and also enhance personal response in reducing exposure to pollutants. There is also need for further research on community perceptions to help understand factors shaping people's perceptions.

\section{Acknowledgements}

We acknowledge the support from Amsterdam Institute for Global Health and Development (AIGHD) for the SCALE-UP study in which this study was nested. This work also used GIS data collected under Nairobi Urban Health and Demographic Surveillance System (NUHDSS). This work was partly undertaken within the Umeå Centre for Global Health Research, with support from FAS, the Swedish Council for Working Life and Social Research (grant No. 2006-1512). We acknowledge the contributions of Alex Ezeh and Eliya Zulu to the conceptualization of the NUHDSS. We also thank those who participated in the study and field workers of SCALE-UP project.

\section{Conflicts of Interest}

The authors declare no conflicts of interest.

\section{References}

1. Bickerstaff, K.; Walker, G. Public understandings of air pollution: The "localisation" of environmental risk. Global Environ. Change 2001, 11, 133-145.

2. Leem, J.H.; Kaplan, B.M.; Shim, Y.K.; Pohl, H.R.; Gotway, C.A.; Bullard, S.M.; Rogers, J.F.; Smith, M.M.; Tylenda, C.A. Exposures to air pollutants during pregnancy and preterm delivery. Environ. Health Perspect. 2006, 114, 905-910.

3. Pope, C.A., III; Dockery, D.W. Health effects of fine particulate air pollution: Lines that connect. J. Air Waste Manag. Assoc. 2006, 56, 709-742. 
4. Pope, C.A., III. Mortality effects of longer term exposures to fine particulate air pollution: Review of recent epidemiological evidence. Inhal. Toxicol. 2007, 19, 33-38.

5. World Health Organization. Air Quality and Health. Available online: http://www.who.int/ mediacentre/factsheets/fs313/en/ (accessed on 17 September 2013).

6. Bell, M.L.; O’Neill, M.S.; Cifuentes, L.A.; Braga, A.L.F.; Green, C.; Nweke, A.; Rogat, J.; Sibold, K. Challenges and recommendations for the study of socioeconomic factors and air pollution health effects. Environ. Sci. Policy 2005, 8, 525-533.

7. Kim, M.; Yi, O.; Kim, H. The role of differences in individual and community attributes in perceived air quality. Sci. Total Environ. 2012, 425, 20-26.

8. World Health Organization. Indoor Air Pollution and Health. Available online: http://www.who.int/ mediacentre/factsheets/fs292/en/index.html/ (accessed on 17 September 2013).

9. Clancy, L.; Goodman, P.; Sinclair, H.; Dockery, D.W. Effect of air-pollution control on death rates in Dublin, Ireland: An intervention study. Lancet 2002, 360, 1210-1214.

10. Parker, J.D.; Mendola, P.; Woodruff, T.J. Preterm birth after the utah valley steel mill closure: A natural experiment. Epidemiology 2008, 19, 820-823.

11. Laden, F.; Schwartz, J.; Speizer, F.E.; Dockery, D.W. Reduction in fine particulate air pollution and mortality: Extended follow-up of the harvard six cities study. Am. J. Respir. Crit. Care Med. 2006, 173, 667-672.

12. Pope, C.A., III; Ezzati, M.; Dockery, D.W. Fine-particulate air pollution and life expectancy in the united states: The role of influential observations. J. Air Waste Manag. Assoc. 2009, 360, 376-386.

13. Giles, L.V.; Barn, P.; Künzli, N.; Romieu, I.; Mittleman, M.A.; ven Eeden, S.; Allen, R.; Carlsten, C.; Stieb, D.; Noonan, C.; et al. From good intentions to proven interventions: Effectiveness of actions to reduce the health impacts of air pollution. Environ. Health Perspect. 2011, 119, 29-36.

14. National Environmental Management Authority-Kenya (NEMA). Highlights on Draft Air Quality Regulations. Available online: http://www.nema.go.ke/index.php?option=com_content\&view= article\&id=141\&Itemid=493/ $($ accessed on 17 September 2013).

15. Ballard-Tremmer, G.; Mathee, A. Review of Interventions to Reduce Exposure of Women and Young Children to Indoor Air Pollution in Developing Countries; WHO/USAID Global Consultation: Washington, DC, USA, 2000.

16. Barnes, B.R.; Mathee, A.; Shafritz, L.B.; Krieger, L.; Zimicki, S. A behavioral intervention to reduce child exposure to indoor air pollution: Identifying possible target behaviors. Health Educ. Behav. 2004, 31, 306-317.

17. Von Schirnding, Y.; Bruce, N.; Smith, K.; Ballard-Tremeer, G.; Ezzati, M.; Lvovsky, K. Addressing the Impact of Household Energy and Indoor Air Pollution on the Health of the Poor: Implications for Policy Action and Intervention Measures. Available online: http://www.who.int/ mediacentre/events/H\&SD_Plaq_no9.pdf (accessed on 17 September 2013).

18. Berry, P.; Clarke, K.; Pajot, M.; Hutton, D.; Verret, M. The Role of Rosk Perception and Health Communication in Adapting to the Health Impacts of Climate Change in Canada. Available online: http://www.climateaccess.org/sites/default/files/Berry_The\%20Role\%20of\%20Risk\%20Perce ption\%20and\%20Health\%20Communication.pdf (accessed on 17 September 2013).

19. Elliott, S.J.; Cole, D.C.; Krueger, P.; Voorberg, N.; Wakefield, S. The power of perception: Health risk attributed to air pollution in an urban industrial neighbourhood. Risk Anal. 1999, 19, 621-634. 
20. Hillier, D. Communicating Health Risks to the Public: A Global Perspective; Gower: Aldershot, UK, 2006.

21. Sjöberg, L.; Moen, B.E.; Rundmo, T. Explaining Risk Perception. An Evaluation of the Psychometric Paradigm in Risk Perception Research; Norwegian University of Science and Technology: Trondheim, Norway, 2004; pp. 1-33.

22. Wakefield, S.E.; Elliott, S.J.; Eyles, J.D.; Cole, D.C. Taking environmental action: The role of local composition, context, and collective. Environ. Manage. 2006, 37, 40-53.

23. Claeson, A.S.; Nordin, M.; Liden, E.; Nordin, S. The role of perceived pollution and health risk perception in annoyance and health symptoms: A population-based study of odorous air pollution. Int. Arch. Occup. Environ. Health 2012, 83, 367-374.

24. Baird, J.C.; Berglund, B.; Berglund, U.; Lindvall, T. Symptom patterns as an early warning signal of community health. Environ. Int.1990, 16, 3-9.

25. Van Thriel, C.; Kiesswetter, E.; Schaper, M.; Juran, S.A.; Blaszkewicz, M.; Kleinbeck, S. Odor annoyance of environmental chemicals: Sensory and cognitive influences. J. Toxicol. Environ. Health 2008, 71, 776-785.

26. Saksena, S. Public Perceptions of Urban Air Pollution with a Focus on Developing Countries; East-West Center: Honolulu, HI, USA, 2007.

27. Gulis, G.; Mulumba, J.A.; Juma, O.; Kakosova, B. Health status of people of slums in Nairobi, Kenya. Environ. Res. 2004, 96, 219-227.

28. Kyobutungi, C.; Ziraba, A.K.; Ezeh, A.; Ye, Y. The burden of disease profile of residents of Nairobi's slums: Results from a demographic surveillance system. Popul. Health Metr. 2008, 6, 1, doi:10.1186/1478-7954-6-1.

29. Egondi, T.; Kyobutungi, C.; Kovats, S.; Muindi, K.; Ettarh, R.; Rocklöv, J. Time-series analysis of weather and mortality patterns in Nairobi's informal settlements. Glob. Health Action. 2012, 5, 23-32.

30. Mutisya, M.; Orindi, B.; Emina, J.; Zulu, E.; Ye, Y. Is mortality among under-five children in nairobi slums seasonal? Trop. Med. Int. Health 2010, 15 132-139.

31. Ye, Y.; Zulu, E.; Mutisya, M.; Orindi, B.; Emina, J.; Kyobutungi, C. Seasonal pattern of pneumonia mortality among under-five children in Nairobi's informal settlements. Am. J. Trop. Med. Hyg. 2009, 81, 770-775.

32. Oti, S.; van de Vijver, S.; Kyobutungi, C.; Gomez, G.B.; Agyemang, C.; van Charante, E.P.M.; Brewster, L.M.; Hendriks, M.E.; Schultsz, C.; Ettarh, R.; et al. Sustainable Model for Cardiovascular Health by Adjusting Lifestyle and Treatment With Economic Perspective in Settings of Urban Poverty-Development, Implementation and Evaluation of a Comprehensive Intervention Package for Primary Prevention of Cardiovascular Diseases in The Slums of Nairobi. Available online: http://controlled-trials.com/ISRCTN84424579 (accessed on 17 September 2013).

33. Emina, J.; Beguy, D.; Zulu, E.M.; Ezeh, A.C.; Muindi, K.; Elung'ata, P.; Otsola, J.K.; Ye, Y. Monitoring of health and demographic outcomes in poor urban settlements: Evidence from the Nairobi urban health and demographic surveillance system. J. Urban Health 2011, 88, S200-S218.

34. Atari, D.O.; Luginaah, I.N.; Fung, K. The relationship between odour annoyance scores and modelled ambient air pollution in Sarnia, "Chemical Valley", Ontario. Int. J. Environ. Res. Public Health 2009, 6, 2655-2675. 
35. Oglesby, L.; Kunzli, N.; Monn, C.; Schindler, C.; Ackermann-Liebrich, U.; Leuenberger, P. Validity of annoyance scores for estimation of long term air pollution exposure in epidemiologic studies: The swiss study on air pollution and lung diseases in adults (SAPALDIA). Am. J. Epidemiol. 2000, 152, 75-83.

36. Bickerstaff, K.; Walker, G. Clearing the smog? Public responses to air-quality information. Local Environ. 1999, 4, 279-294.

37. Howel, D.; Moffatt, S.; Bush, J.; Dunn, C.E.; Prince, H. Public views on the links between air pollution and health in northeast england. Environ. Res. 2003, 91, 163-171.

38. Jacobi, P.R. Households and environment in the city of São Paulo; problems, perceptions and solutions. Environ. Urban. 1994, 6, 87-110.

39. Jacquemin, B.; Sunyer, J.; Forsberg, B.; Götschi, T.; Bayer-Oglesby, L.; Ackermann-Liebrich, U.; de Marco, R.; Heinrich, J.; Jarvis, D.; Torén, K.; et al. Annoyance due to air pollution in europe. Int. J. Epidemiol.2007, 36, 809-820.

40. Klaeboe, R.; Kolbenstvedt, M.; Clench-Aas, J.; Bartonova, A. Oslo traffic study-Part 1: An integrated approach to assess the combined effects of noise and air pollution on annoyance. Atmos. Environ. 2000, 34, 4727-4736.

41. Semenza, J.C.; Wilson, D.J.; Parra, J.; Bontempo, B.D.; Hart, M.; Sailor, D.J.; George, L.A. Public perception and behavior change in relationship to hot weather and air pollution. Environ. Res. 2008, 107, 401- 411.

42. Brody, S.D.; Peck, B.M.; Highfield, W.E. Examining localized patterns of air quality perception in Texas: A spatial and statistical analysis. Risk Anal. 2004, 24, 1561-1574.

43. Fischer, G.; Morgan, M.; Fischhoff, B.; Nair, I.; Lave, L. What risks are people concerned about. Risk Anal. 1991, 11, 303-314.

44. Brown, M.; Macey, S. Understanding residential energy-conservation through attitudes and beliefs. Environ. Plan. A 1983, 15, 405-416.

45. Kohlhuber, M.; Mielck, A.; Weiland, S.K.; Bolte, G. Social inequality in perceived environmental exposures in relation to housing conditions in Germany. Environ. Res. 2006, 101, 246-255.

46. Lercher, P.; Schmitzberger, R.; Kofler, W. Perceived traffic air pollution, associated behavior and health in an alpine area. Sci. Total Environ.1995, 169, 71-74.

47. Wallner, A.; Hunziker, M.; Kienast, F. Do natural science experiments influence public attitudes towards environmental problems? Global Environ. Change 2003, 13, 185-194.

(C) 2013 by the authors; licensee MDPI, Basel, Switzerland. This article is an open access article distributed under the terms and conditions of the Creative Commons Attribution license (http://creativecommons.org/licenses/by/3.0/). 